\title{
Infinite words containing squares at every position
}

\author{
James Currie*and Narad Rampersad ${ }^{\dagger}$ \\ Department of Mathematics and Statistics \\ University of Winnipeg \\ 515 Portage Avenue \\ Winnipeg, Manitoba R3B 2E9 (Canada) \\ j.currie@uwinnipeg.ca \\ n.rampersad@uwinnipeg.ca
}

August 13, 2021

\begin{abstract}
Richomme asked the following question: what is the infimum of the real numbers $\alpha>2$ such that there exists an infinite word that avoids $\alpha$-powers but contains arbitrarily large squares beginning at every position? We resolve this question in the case of a binary alphabet by showing that the answer is $\alpha=7 / 3$.
\end{abstract}

\section{Introduction}

We consider the following question of Richomme [17]: what is the infimum of the real numbers $\alpha>2$ such that there exists an infinite word that avoids $\alpha$-powers but contains arbitrarily large squares beginning at every position? As we shall see, over the binary alphabet, the answer to Richomme's question is $\alpha=7 / 3$.

First we recall some basic definitions. If $\alpha$ is a rational number, a word $w$ is an $\alpha$-power if there exist words $x$ and $x^{\prime}$, with $x^{\prime}$ a prefix of $x$, such that $w=x^{n} x^{\prime}$ and $\alpha=n+\left|x^{\prime}\right| /|x|$. We refer to $|x|$ as a period of $w$. An $\alpha^{+}$-power is a word that is a $\beta$-power for some $\beta>\alpha$. A word is $\alpha$-power-free (resp. $\alpha^{+}$-power-free) if none of its subwords is an $\alpha$-power (resp. $\alpha^{+}$-power). A 2-power is called a square; a $2^{+}$-power is called an overlap.

The motivation for Richomme's question comes from the observation that there exist aperiodic infinite binary words that contain arbitrarily large squares starting at every position. For instance, all Sturmian words have this property [1, Proposition 2].

${ }^{*}$ The author is supported by an NSERC Discovery Grant.

†The author is supported by an NSERC Postdoctoral Fellowship. 
Certain Sturmian words are not only aperiodic, but also avoid $\alpha$-powers for some real number $\alpha$. For instance, it is well-known that the Fibonacci word

$$
\mathbf{f}=010010100100101001010 \cdots
$$

contains no $(2+\varphi)$-powers [14] (see also [13]), where $\varphi=(1+\sqrt{5}) / 2$ is the golden ratio. By contrast, the Thue-Morse word

$$
\mathbf{t}=011010011001011010010110 \cdots
$$

is overlap-free [20] but does not contain squares beginning at every position (It is an easy exercise to show that $\mathbf{t}$ does not begin with a square). The squares occurring in the ThueMorse word have been characterized by Pansiot [15] and Brlek [4, and the positions at which they occur were studied by Brown et al. [5].

Saari [18] also studied infinite words containing squares (not necessarily arbitrarily large) beginning at every position. He calls such words squareful (though he imposes the additional condition that the word contain only finitely many distinct minimal squares).

\section{Overlap-free and 7/3-power-free squares}

We begin by reviewing what is known concerning the overlap-free binary squares. Subsequently, we shall generalize this characterization to the $7 / 3$-power-free binary squares.

Let $\mu$ denote the Thue-Morse morphism: i.e., the morphism that maps $0 \rightarrow 01$ and $1 \rightarrow 10$. Define sets

$$
A=\{00,11,010010,101101\}
$$

and

$$
\mathcal{A}=\bigcup_{k \geq 0} \mu^{k}(A)
$$

The set $\mathcal{A}$ is the set of squares appearing in the Thue-Morse word. Shelton and Soni [19] characterized the overlap-free squares (the result is also attributed to Thue by Berstel [2]), as being the conjugates of the words in $\mathcal{A}$ (A conjugate of $x$ is a word $y$ such that $x=u v$ and $y=v u$ for some $u, v)$. An immediate application is the following theorem:

Theorem 1. If $\mathbf{w}$ is an infinite overlap-free binary word, then there is a position $i$ such that $\mathbf{w}$ does not contain a square beginning at position $i$.

Proof. An easy computer search suffices to verify that any overlap-free word over $\{0,1\}$ of length greater than 36 must contain the subword 010011. Let $i$ denote any position at which 010011 occurs in $\mathbf{w}$. We claim that no square begins at position $i$. Suppose to the contrary that $x x$ is such a square. By Shelton and Soni's result, except for the squares that are conjugates of $A$, every overlap-free square $x x$ has $|x|$ even. It follows that $x x$ is of the form $0 y 10 y 1$, where $y \in\{01,10\}^{\ell}$ for some $\ell$. However, this forces $x x$ to be followed by 0 in $\mathbf{w}$, so that we have the overlap $x x 0$ as a subword of $\mathbf{w}$, a contradiction. 
The next theorem generalizes the characterization of Shelton and Soni.

Theorem 2. The 7/3-power-free binary squares are the conjugates of the words in $\mathcal{A}$.

We defer the proof of Theorem 2 to Section 4 . However, we end this section by proving an analogue, for 7/3-power-free words, of a well-known "progression lemma" for overlap-free words [3, 7, 10, 11, 16, 19] that we shall need later.

The words $\mu^{n}(0)$ and $\mu^{n}(1), n \geq 0$, are known as Morse blocks. Note that the reverse of a Morse block is a Morse block.

Lemma 3. Let $w=u v x y$ be a binary 7/3-power-free word with $|u|=|v|=|x|=|y|=2^{n}$. If $u$ and $v$ are Morse blocks, then $x$ is a Morse block.

Proof. The proof is by induction on $n$. Clearly, the result holds for $n=0$. We have either (1) $w=u^{\prime} u^{\prime \prime} u^{\prime} u^{\prime \prime}$ pqrs or (2) $w=u^{\prime} u^{\prime \prime} u^{\prime \prime} u^{\prime}$ pqrs, where $u^{\prime}$ and $u^{\prime \prime}$ are distinct Morse blocks of length $2^{n-1}$ and $|p|=|q|=|r|=|s|=2^{n-1}$. By induction, $p, q$, and $r$ are also Morse blocks. We must show that $p \neq q$. In case (1), $p q=u^{\prime} u^{\prime}$ creates the $5 / 2$-power $u^{\prime} u^{\prime \prime} u^{\prime} u^{\prime \prime} u^{\prime}$, and $p q=u^{\prime \prime} u^{\prime \prime}$ creates the cube $u^{\prime \prime} u^{\prime \prime} u^{\prime \prime}$. In case (2), $p q=u^{\prime} u^{\prime}$ creates the cube $u^{\prime} u^{\prime} u^{\prime}$; and if $p q=u^{\prime \prime} u^{\prime \prime}$, then $r=u^{\prime}$ creates the $7 / 3$-power $u^{\prime} u^{\prime \prime} u^{\prime \prime} u^{\prime} u^{\prime \prime} u^{\prime \prime} u^{\prime}$, and $r=u^{\prime \prime}$ creates the cube $u^{\prime \prime} u^{\prime \prime} u^{\prime \prime}$. Thus, $p \neq q$, as required.

\section{Infinite words containing squares at every position}

We begin by showing that the answer to Richomme's question is at most $7 / 3$.

Theorem 4. There exists an infinite $(7 / 3)^{+}$-power-free binary word that contains arbitrarily large squares beginning at every position.

Proof. We rely on the existence of a $(7 / 3)^{+}$-power-free morphism; that is, a morphism $f$ such that $f(w)$ is $(7 / 3)^{+}$-power-free whenever $w$ is $(7 / 3)^{+}$-power-free. Kolpakov, Kucherov, and Tarannikov [12] have given an example of such a morphism $f$ :

$$
\begin{aligned}
& 0 \rightarrow 011010011001001101001 \\
& 1 \rightarrow 100101100100110010110 .
\end{aligned}
$$

For convenience we work instead with the following morphism $g$ :

$$
\begin{aligned}
& 0 \rightarrow 011010011011001101001 \\
& 1 \rightarrow 100101100110110010110
\end{aligned}
$$

which we obtain by setting $g(0)=\overline{f(1)}$ and $g(1)=\overline{f(0)}$, where the overline denotes binary complementation.

We now define a sequence of words $\left(A_{n}\right)_{n \geq 0}$ as follows. Let $A_{0}=0110110$. For $n \geq 0$ define $A_{n+1}=(011010)^{-1} g\left(A_{n}\right)$, where the notation $(011010)^{-1} x$ denotes the word obtained 
by removing the prefix 011010 from the word $x$. Since $g\left(A_{n}\right)$ always begins with 011010, this operation is well-defined. The sequence $\left(A_{n}\right)_{n \geq 0}$ thus begins

$$
\begin{aligned}
& A_{0}=0110110 \\
& A_{1}=011011001101001100101100110110010110100101100110110010110 \ldots
\end{aligned}
$$

Observe that since $g$ is $(7 / 3)^{+}$-power-free, $A_{n}$ is also $(7 / 3)^{+}$-power-free. We first show that as $n \rightarrow \infty, A_{n}$ converges to an infinite limit word $\mathbf{w}$; second, we show that $\mathbf{w}$ contains arbitrarily long squares beginning at every position.

We show by induction on $n$ that $A_{n}$ is a prefix of $A_{n+1}$. Clearly this is true for $n=0$. Recall that $A_{n+1}=(011010)^{-1} g\left(A_{n}\right)$. Inductively, $A_{n-1}$ is a prefix of $A_{n}$, so $g\left(A_{n-1}\right)$ is a prefix of $g\left(A_{n}\right)$. Thus, $(011010)^{-1} g\left(A_{n-1}\right)=A_{n}$ is a prefix of $(011010)^{-1} g\left(A_{n}\right)=A_{n+1}$, as required. We conclude that $A_{n}$ tends, in the limit, to a $(7 / 3)^{+}$-power-free word $\mathbf{w}$.

To see that $\mathbf{w}$ contains arbitrarily long squares beginning at every position, first let $u=011010$ and observe that for every $n \geq 1$ we have

$$
A_{n}=u^{-1} g(u)^{-1}\left[g^{2}(u)\right]^{-1} \cdots\left[g^{n-1}(u)\right]^{-1} g^{n}(0110110) .
$$

Let

$$
v=g^{n-1}(u) g^{n-2}(u) \cdots g(u) u,
$$

so that $A_{n}=v^{-1} g^{n}(0110110)$, and observe that

$$
|v|=6 \sum_{j=0}^{n-1} 21^{j}=6\left(\frac{21^{n}-1}{21-1}\right)<21^{n}=\left|g^{n}(0)\right| .
$$

We see then that $v$ is a prefix of $g^{n}(0)$. Write $g^{n}(0)=v x$, so that $A_{n}=x g^{n}(11) v x g^{n}(11) v x$. It follows that for $0 \leq j<|x|=21^{n}-(3 / 10)\left(21^{n}-1\right), A_{n}$, and hence $\mathbf{w}$, contains a square of length $6 \cdot 21^{n}$ beginning at position $j$. Since $n$ may be taken to be arbitrarily large, the result follows.

The result of the previous theorem is optimal, as we now demonstrate.

Theorem 5. If $\mathbf{w}$ is an infinite 7/3-power-free binary word, then there is a position $i$ such that $\mathbf{w}$ does not contain arbitrarily large squares beginning at position $i$.

Proof. As in the proof of Theorem 1, an easy computer search suffices to verify that any $7 / 3$-power-free word over $\{0,1\}$ of length greater than 39 must contain the subword 010011. Let $i$ be a position at which there is an occurrence of 010011 in $\mathbf{w}$. Suppose that there is a square $x x$ beginning at position $i$. By Theorem 2, $x x$ is a conjugate of a word in $\mathcal{A}$. In particular, $x x \notin \mathcal{A}$; that is, $x x$ is a non-identity conjugate of a word in $\mathcal{A}$.

Case 1: $x x$ is a conjugate of either $\mu^{k}(00)$ or $\mu^{k}(11)$ for some $k$. Then $x x$ is a conjugate of a word of the form uvuv, where $u$ and $v$ are Morse blocks of the same length. Without loss of generality, we write $x x=u^{\prime \prime} v u v u^{\prime}$, where $u^{\prime} u^{\prime \prime}=u$ and $u^{\prime} \neq \epsilon \neq u^{\prime \prime}$. 
Suppose that $y y$ is another square beginning at position $i$. Suppose further that there are arbitrarily large squares beginning at position $i$, so that we may choose $|y|>|x x|$. We see then that there is an occurrence of $u^{\prime \prime} v u v u^{\prime}$ at position $i+|y|$. Considering this later occurrence of $u^{\prime \prime} v u v u^{\prime}$, and observing that Morse blocks of a given length are uniquely identified by their first letter (as well as by their last letter), we may apply Lemma 3 to this later occurrence of $u^{\prime \prime} v u v u^{\prime}$ to conclude that the $v u v$ of this occurrence is both preceded and followed by the Morse block $u$. Thus, w contains the 5/2-power uvuvu, a contradiction.

Case 2: $x x$ is a conjugate of either $\mu^{k}(010010)$ or $\mu^{k}(101101)$ for some $k$. By a similar argument as in Case 1, we may suppose that $x x$ has one of the forms $u^{\prime \prime} v u u v u u^{\prime}, u^{\prime \prime} v v u v v u^{\prime}$, or $u^{\prime \prime} u v u u v u^{\prime}$, where $u$ and $v$ are Morse blocks of the same length, $u^{\prime} u^{\prime \prime}=u$, and $u^{\prime} \neq \epsilon \neq u^{\prime \prime}$.

As before, we suppose the existence of a square $y y$ beginning at position $i$, where $|y|>$ $|x x|$. Then there is a later occurrence of $x x$ at position $i+|y|$. Applying Lemma 3 to this later occurrence of $x x$, we deduce the existence of one of the $7 / 3$-powers uvuuvuu, uvvuvvu, or uuvuuvu, a contradiction.

All cases yield a contradiction; we conclude that there does not exist arbitrarily large squares beginning at position $i$, as required.

It is possible, however, to have an infinite $7 / 3$-power-free binary word with squares beginning at every position; we are only prevented from having arbitrarily large squares beginning at every position.

Theorem 6. There exists an infinite 7/3-power-free binary word that contains squares beginning at every position.

Proof. We show that a word constructed by Currie, Rampersad, and Shallit [6] has the desired property. The construction is as follows. We define the following sequence of words: $A_{0}=00$ and $A_{n+1}=0 \mu^{2}\left(A_{n}\right), n \geq 0$. The first few terms in this sequence are

$$
\begin{aligned}
& A_{0}=00 \\
& A_{1}=001100110 \\
& A_{2}=0011001101001100101100110100110010110
\end{aligned}
$$

Currie et al. showed that as $n \rightarrow \infty$, this sequence converges to an infinite word $\mathbf{a}$, and further, $\mathbf{a}$ is $7 / 3$-power-free. We show that a contains squares beginning at every position. We claim that for $n \geq 0$, a contains a word of the form $x x x^{\prime}$ at position $\left(4^{n}-1\right) / 3$, where $|x|=4^{n+1}$ and $x^{\prime}$ is a prefix of $x$ of length $4^{n}$. Observe that for $n \geq 0$, by the construction of $A_{n+1}$, we have

$$
A_{n+1}=0 \mu^{2}(0) \mu^{4}(0) \cdots \mu^{2(n-1)}(0) \mu^{2 n}\left(A_{1}\right) .
$$

However, $\mu^{2 n}\left(A_{1}\right)=x x x^{\prime}$, where $x=\mu^{2 n}(0011)$ has length $4^{n+1} ; x^{\prime}=\mu^{2 n}(0)$ is a prefix of $x$ of length $4^{n}$; and $\mu^{2 n}\left(A_{1}\right)$ occurs at position

$$
\sum_{i=0}^{n-1} 4^{i}=\frac{4^{n}-1}{3}
$$


as claimed. It follows that for $i \in\left[\left(4^{n}-1\right) / 3,\left(4^{n+1}-1\right) / 3-1\right]$, a contains a square of length $2 \cdot 4^{n+1}$ at position $i$. This completes the proof.

Although the word constructed in the proof of Theorem [6] contains squares beginning at every position, it is not squareful in the sense of Saari [18], since it does not contain only finitely many minimal squares. For a squareful word, there exists a constant $C$ such that at every position there is a square of length at most $C$. To see that this does not hold for the word a constructed above, we note that by the factorization theorem of Karhumäki and Shallit [9] (Theorem 8 below), any infinite 7/3-power-free binary word contains occurrences of $\mu^{n}(0)$ for arbitrarily large $n$. However, $\mu^{n}(0)$ is a prefix of the Thue-Morse word, and we have already noted in the introduction that the Thue-Morse word does not begin with a square. Thus there cannot exist a constant $C$ bounding the length of a minimal square in $\mathbf{a}$, so $\mathbf{a}$ is not squareful. In general, no infinite $7 / 3$-power-free binary word can be squareful.

The result of Theorem [ can be strengthened by applying a more general construction of Currie et al. [6].

Theorem 7. For every real number $\alpha>2$, there exists an infinite $\alpha$-power-free binary word that contains squares beginning at every position.

Proof. Since Theorem [6 establishes the result for $\alpha \geq 7 / 3$, we only consider $\alpha<7 / 3$. We recall the following construction of Currie et al. [6, Theorem 14]. Let $s \geq 3$ and $t \geq 5$ be integers such that $2<3-t / 2^{s}<\alpha$, and such that the word obtained by removing the prefix of length $t$ from $\mu^{s}(0)$ begins with 00 . Let $\beta=3-t / 2^{s}$.

We construct sequences of words $A_{n}, B_{n}$ and $C_{n}$. Define $C_{0}=00$ and let $u$ be the prefix of length $t$ of $\mu^{s}(0)$. For each $n \geq 0$ :

1. Let $A_{n}=0 C_{n}$.

2. Let $B_{n}=\mu^{s}\left(A_{n}\right)$.

3. Let $C_{n+1}=u^{-1} B_{n}$.

Currie et al. showed that the $C_{n}$ 's converge to an infinite word $\mathbf{w}$ that is $\beta^{+}$-power-free, and hence, $\alpha$-power-free. For $n \geq 1$, w begins with a prefix of the form

$$
u^{-1} \mu^{s}(0)\left[\mu^{s}(u)\right]^{-1} \mu^{2 s}(0) \cdots\left[\mu^{n s}(u)\right]^{-1} \mu^{(n+1) s}(0) \mu^{(n+1) s}(00) .
$$

Let $u^{\prime}=u^{-1} \mu^{s}(0)$. Thus, for $n \geq 1$, w contains the word $\mu^{n s}\left(u^{\prime}\right) \mu^{(n+1) s}(00)$ at position

$$
F_{n}=2^{s} \sum_{i=0}^{n-1} 2^{i s}-t \sum_{i=0}^{n-1} 2^{i s}=\left(2^{s}-t\right)\left[\frac{2^{n s}-1}{2^{s}-1}\right]
$$

where $\mu^{n s}\left(u^{\prime}\right)$ is a suffix of $\mu^{(n+1) s}(0)$. Letting $\left|u^{\prime}\right|=t^{\prime}$ and defining $G_{n}=\left|\mu^{n s}\left(u^{\prime}\right)\right|=t^{\prime} \cdot 2^{n s}$, we have

$$
F_{n}=t^{\prime}\left[\frac{2^{n s}-1}{2^{s}-1}\right]<G_{n} .
$$


Since $u^{\prime}$ is a suffix of $\mu^{s}(0)$, and since $\mathbf{w}$ begins with $u^{\prime} \mu^{s}(00)$, we see that for $j \in\left[0, t^{\prime}-1\right]$, every subword of $\mathbf{w}$ of length $2^{s+1}$ starting at position $j$ is a square. Similarly, for $n \geq 1$, we see that there is a square of length $2^{(n+1) s+1}$ starting at position $j$ for every $j \in\left[F_{n}, F_{n}+\right.$ $\left.G_{n}-1\right]$. Since $F_{n}<G_{n}$, there is thus a square at every position of $\mathbf{w}$.

\section{Proof of Theorem 2}

In this section we give the proof of Theorem 2. We begin with some lemmas, but first we recall the factorization theorem of Karhumäki and Shallit [9].

Theorem 8 (Karhumäki and Shallit). Let $x \in\{0,1\}^{*}$ be $\alpha$-power-free, $2<\alpha \leq 7 / 3$. Then there exist $u, v \in\{\epsilon, 0,1,00,11\}$ and an $\alpha$-power-free $y \in\{0,1\}^{*}$ such that $x=u \mu(y) v$.

Lemma 9. Let $x x \in\{0,1\}^{*}$ be $7 / 3$-power-free. If $x x=\mu(y)$, then $|y|$ is even. Consequently, $y$ is a square.

Proof. Suppose to the contrary that $|y|=|x|$ is odd. By an exhaustive enumeration one verifies that $|x| \geq 5$. But then $x x$ contains two occurrences of 00 (or 11) in positions of different parities, which is impossible.

The next lemma is a version of Theorem 8 specifically applicable to squares.

Lemma 10. Let $x x \in\{0,1\}^{*}$ be $7 / 3$-power-free. If $|x x|>8$, then either

(a) $x x=\mu(y)$, where $y \in\{0,1\}^{*}$; or

(b) $x x=\bar{a} \mu(y) a$, where $a \in\{0,1\}$ and $y \in\{0,1\}^{*}$.

Proof. Applying Theorem 8, we write $x x=u \mu(y) v$. We first show that $|u|=|v| \leq 1$. Suppose that $u=00$. Then $x x$ begins with one of the words 000, 00100, or 001010. The first and third words contain a 7/3-power, a contradiction. The second word, 00100, cannot occur later in $x x$, as that would also imply the existence of a $7 / 3$-power. We conclude $u \neq 00$, and similarly, $u \neq 11$. A similar argument also holds for $v$. Since $|x x|$ is even, we must therefore have $|u|=|v|$, as required.

If $|u|=|v|=0$, then we have established (a). If $|u|=|v|=1$, it remains to show that $u=\bar{v}$. If $u=v$, then we have $x x=u \mu(y) u$. Since $x$ begins and ends with $u$, we have $x=u \mu\left(y^{\prime}\right)=\mu\left(y^{\prime \prime}\right) u$, where $y=y^{\prime} y^{\prime \prime}$. Let $z^{\prime}=\mu\left(y^{\prime}\right)$ and $z^{\prime \prime}=\mu\left(y^{\prime \prime}\right)$. Then $z^{\prime \prime}$ begins with $u$ and hence with $u \bar{u}$. This implies that $z^{\prime}$ begins with $\bar{u}$ and hence with $\bar{u} u$. This in turn implies that $z^{\prime \prime}$ begins with $u \bar{u} u \bar{u}$, and hence that $z^{\prime}$ begins with $\bar{u} u \bar{u} u$. Now we see that $x$ begins with the $5 / 2$-power $u \bar{u} u \bar{u} u$, which is a contradiction. We conclude that $u \neq v$, as required.

We are now ready to prove Theorem 2 . 
Proof of Theorem 2. Let $x x$ be a minimal 7/3-power-free square that is not a conjugate of a word in $\mathcal{A}$. That $|x x|>8$ is easily verified computationally. Applying Lemma 10 leads to two cases.

Case 1: $x x=\mu(y)$. By Lemma 9, $y$ is a square. Furthermore, $y$ is not a conjugate of a word in $\mathcal{A}$, contradicting the minimality of $x x$.

Case 2: $x x=\bar{a} \mu(y) a$. Then $a \bar{a} \mu(y)=\mu(a y)$ is also a square $z z$. We show that $z z$ is $7 / 3$ power-free, and consequently, by Lemma 9 , that ay is a $7 / 3$-power-free square, contradicting the minimality of $x x$.

Suppose to the contrary that $z z$ contains a $7 / 3$-power $s=r r r^{\prime}$, where $r^{\prime}$ is a prefix of $r$ and $\left|r^{\prime}\right| /|r| \geq 1 / 3$. The word $s$ must occur at the beginning of $z z$ and we must have $|s|>|x|$; otherwise, $x x$ would contain an occurrence of $s$, contradicting the assumption that $x x$ is $7 / 3$-power-free. We have four cases, depending on the relative sizes of $|r|$ and $|z|$, as illustrated in Figures 14 .

By analyzing the overlaps between $z z$ and $r r r^{\prime}$, denoted $X$ in the figures, we derive a contradiction in each case.

Case 2a: $2|r|<|z| \leq 2|r|+\left|r^{\prime}\right| / 2$ (Figure 1). In this case, $r^{\prime}$ has a prefix $X$ that is also a suffix of $z$. Then $X$ is also a prefix of $z$ and $X X$ is a prefix of $r^{\prime}$. Consequently, $X X$ is a prefix of $z$ and $x x$ contains the cube $X X X$. This is a contradiction.

Case 2b: $|z|>2|r|+\left|r^{\prime}\right| / 2$ (Figure 2). In this case, $r^{\prime}$ is of the form $X Y X$, where $X Y$ is a suffix of $z$ and $X$ is a prefix of $z$. Since $r^{\prime}=X Y X$, then $z$ has $X Y X$ as a prefix. In particular, $x x$ contains a $7 / 3$-power-free square $X Y X Y$. By the assumed minimality of $x x$, $X Y X Y$ is a conjugate of a word in $\mathcal{A}$. If $X Y X Y=\mu^{k}(w)$, where $w$ is a conjugate of a word in $A$ (not $\mathcal{A}$ !), then $X Y X Y$ can be written either as $A_{1} A_{1}$ or as $A_{1} A_{2} A_{3} A_{1} A_{2} A_{3}$, where the $A_{i}$ 's are all Morse blocks of the same length. Since $X Y X Y$ is followed by $X$ in $z z$, and since the Morse blocks of a given length are uniquely identified by their first letter, by Lemma 3 $X Y X Y$ is followed by the Morse block $A_{1}$, creating either a cube or a $7 / 3$-power in $x x$, contrary to our assumption.

If $X Y X Y \neq \mu^{k}(w)$, where $w$ is a conjugate of a word in $A$, then we may write $X Y X Y$ as one of $u B A B v, u B A A B A v, u B B A B B v$, or $u A B A A B v$, where $A$ and $B$ are Morse blocks, $B=\bar{A}$, and $v u=A$.

If $X Y X Y=u B A B v$, then, since $u$ is a non-empty suffix of $A$ and $v$ is a non-empty prefix of $A$, and since the Morse blocks of a given length are uniquely identified by their first letter (as well as by their last letter), we can apply Lemma 3 to conclude that $B A B$ is preceded and followed by $A$. Thus $x x$ contains the $5 / 2$-power $A B A B A$, contrary to our assumption. Similarly, if $X Y X Y=u B A A B A v$, then $B A A B A$ is preceded and followed by $A$, creating the $7 / 3$-power $A B A A B A A$. The other possibilities for $X Y X Y$ lead to the existence of a $7 / 3$-power in $x x$ by a similar argument.

Case 2c: $|z| \leq 3 / 2 \cdot|r|$ (Figure 3). In this case, $r$ has a prefix $X$ that is also a suffix of $z$. Then $X$ is also a prefix of $z$ and $X X$ is a prefix of $r$. Consequently, $X X$ is a prefix of $z$ and $x x$ contains the cube $X X X$. This is a contradiction.

Case 2d: $3 / 2 \cdot|r|<|z|<2|r|$ (Figure 4). In this case, $r$ has a suffix $X$ that is also a prefix of $z$. Then $X$ is also a prefix of $r$ and $r^{\prime}$ begins with some prefix $X^{\prime}$ of $X$. Consequently, 


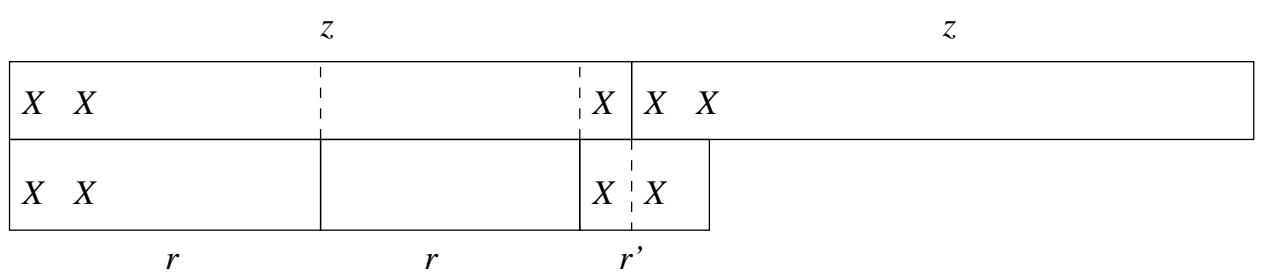

Figure 1: The case where $2|r|<|z| \leq 2|r|+\left|r^{\prime}\right| / 2$

\begin{tabular}{|c|c|c|c|c|}
\hline $\begin{array}{lll}X & Y & X\end{array}$ & & $Y$ & & $Y \quad X$ \\
\hline $\begin{array}{lll}X & Y & X\end{array}$ & $X$ & $Y:$ & $X$ & \\
\hline
\end{tabular}

Figure 2: The case where $|z|>2|r|+\left|r^{\prime}\right| / 2$

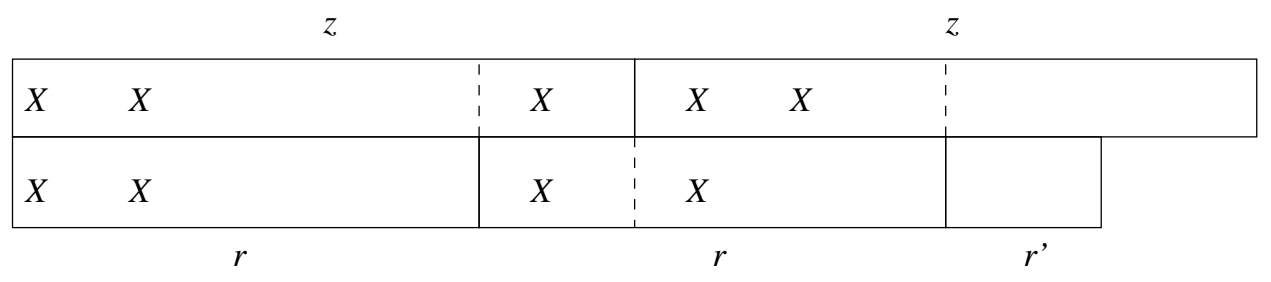

Figure 3: The case where $|z| \leq 3 / 2 \cdot|r|$

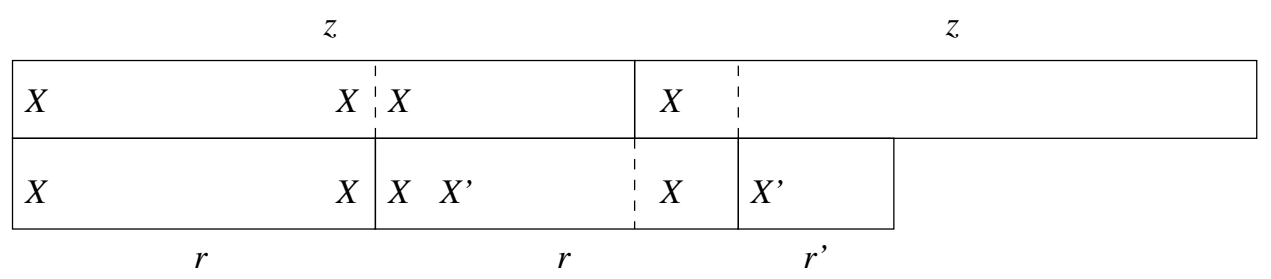

Figure 4: The case where $3 / 2 \cdot|r|<|z|<2|r|$ 
$r r$, and indeed $x x$, contains a subword $X X X^{\prime}$ that is at least a $7 / 3$-power. This is a contradiction.

Since in all cases we have derived a contradiction by showing that $x x$ contains a $7 / 3$ power, we conclude that our assumption that $z z$ contains a $7 / 3$-power is false. Recalling that $z z=\mu(a y)$ and that $a y$ is necessarily a square, we conclude that ay is a $7 / 3$-power-free square, contradicting the minimality of $x x$. We conclude that there exists no $7 / 3$-power-free square $x x$ that is not a conjugate of a word in $\mathcal{A}$.

We claim that the constant $7 / 3$ in Theorem 2 is best possible. To see this, note that the word

$$
01101001101100101100110100110110010110
$$

is a $(7 / 3)^{+}$-power-free square, but is not a conjugate of a word in $\mathcal{A}$.

\section{Conclusion}

We have only considered words over a binary alphabet. It remains to consider whether similar results hold over a larger alphabet. For instance, does there exist an infinite overlapfree ternary word that contains squares beginning at every position? Richomme observes that over any alphabet there cannot exist an infinite overlap-free word containing infinitely many squares at every position. He points out that this follows from the following result of Ilie [8, Lemma 2]: In any word, if $v v$ and $u u$ are two squares at position $i$ and $w w$ is a square at position $i+1$, then either $|w|=|u|$ or $|w|=|v|$ or $|w| \geq 2|v|$. An easy consequence of this result is that in any infinite word, if infinitely many distinct squares begin at position $i$ and $w w$ is a square beginning at position $i+1$, then $|w|=|u|$ for some square $u u$ occurring at position $i$, and hence there is an overlap at position $i$.

\section{Acknowledgments}

The authors wish to thank Gwénaël Richomme for suggesting the problem. We also thank him for reading an earlier draft of this paper and providing many helpful comments and suggestions.

\section{References}

[1] J.-P. Allouche, J. L. Davison, M. Queffélec, L. Q. Zamboni, "Transcendence of Sturmian or morphic continued fractions", J. Number Theory 91 (2001), 39-66.

[2] J. Berstel, "Axel Thue's work on repetitions in words". In P. Leroux, C. Reutenauer, eds., Séries formelles et combinatoire algébrique, Publications du LaCIM, pp 65-80, UQAM, 1992. 
[3] J. Berstel, "A rewriting of Fife's theorem about overlap-free words". In J. Karhumäki, H. Maurer, G. Rozenberg, eds., Results and Trends in Theoretical Computer Science, LNCS 812, pp. 19-29 Springer-Verlag, 1994.

[4] S. Brlek, "Enumeration of factors in the Thue-Morse word", Discrete Appl. Math. 24 (1989), 83-96.

[5] S. Brown, N. Rampersad, J. Shallit, T. Vasiga, "Squares and overlaps in the Thue-Morse sequence and some variants", Theor. Inform. Appl. 40 (2006), 473-484.

[6] J. Currie, N. Rampersad, J. Shallit, "Binary words containing infinitely many overlaps", Electron. J. Combinatorics 13 (2006), \#R82.

[7] E. Fife, "Binary sequences which contain no BBb", Trans. Amer. Math. Soc. 261 (1980), $115-136$.

[8] L. Ilie, "A note on the number of squares in a word", Theoret. Comput. Sci. 380 (2007), $373-376$.

[9] J. Karhumäki, J. Shallit, "Polynomial versus exponential growth in repetition-free binary words", J. Combin. Theory Ser. A 104 (2004), 335-347.

[10] R. Kfoury, "A linear time algorithm to decide whether a binary word contains an overlap", Theoret. Inform. Appl. 22 (1988), 135-145.

[11] Y. Kobayashi, "Enumeration of irreducible binary words", Discrete Appl. Math. 20 (1988), 221-232.

[12] R. Kolpakov, G. Kucherov, Y. Tarannikov, "On repetition-free binary words of minimal density", WORDS (Rouen, 1997), Theoret. Comput. Sci. 218 (1999), 161-175.

[13] D. Krieger, "On critical exponents in fixed points of non-erasing morphisms", Theoret. Comput. Sci. 376 (2007), 70-88.

[14] F. Mignosi, G. Pirillo, "Repetitions in the Fibonacci infinite word", RAIRO Inform. Théor. 26 (1992), 199-204.

[15] J. J. Pansiot, "The Morse sequence and iterated morphisms", Inform. Process. Lett. 12 (1981), 68-70.

[16] A. Restivo, S. Salemi, "Overlap free words on two symbols". In M. Nivat, D. Perrin, eds., Automata on Infinite Words, LNCS 192, pp. 198-206, Springer-Verlag, 1984.

[17] G. Richomme. Personal communication, 2005.

[18] K. Saari, "Everywhere $\alpha$-repetitive sequences and Sturmian words". In Proc. CSR 200\%, LNCS 4649, pp. 362-372, Springer-Verlag, 2007. 
[19] R. Shelton, R. Soni, "Chains and fixing blocks in irreducible sequences", Discrete Math. 54 (1985), 93-99.

[20] A. Thue, "Über die gegenseitige Lage gleicher Teile gewisser Zeichenreihen", Kra. Vidensk. Selsk. Skrifter. I. Math. Nat. Kl. 1 (1912), 1-67. 